\title{
Etude comparative de la nodulation et du rendement de quelques variétés d'arachide (Arachis hypogaea L., Fabaceae) cultivées en conditions éco-climatiques de Kinshasa, République Démocratique du Congo
}

\author{
B.M. BANGATA ${ }^{1 *}$, K.N. NGBOLUA ${ }^{2}$, M. MAWA ${ }^{1}$, M. MINENGU $^{1}$ et \\ K. N. MOBAMBO ${ }^{1}$ \\ ${ }^{1}$ Département de Phytotechnie, Faculté des Sciences Agronomiques, Université de Kinshasa, \\ BP.117 Kinshasa XI, République Démocratique du Congo. \\ ${ }^{2}$ Département de Biologie, Faculté des Sciences, Université de Kinshasa, BP. 190 Kinshasa XI, \\ République Démocratique du Congo. \\ *Auteur correspondant; E-mail : bithanyimbunzu@yahoo.fr; bithanyimbunzu@unikin.ac.cd; \\ Tél. : +243813630475.
}

\section{RESUME}

Une expérimentation à été conduite du 14 Avril au 24 Juillet 2012 sur les cultures légumineuses fixatrices d'azote, en vue de servir d'alternative pour améliorer la nutrition azotée et augmenter les rendements des cultures. Le rapport entre le taux de fixation de l'azote et le rendement en graines a été étudié pour 12 variétés d'arachide dans les conditions éco-climatiques de Kinshasa/Ndjili Brasserie. Cette recherche avait pour objectifs de déterminer l'abondance de la nodulation à 60 jours après le semis et évaluer le rendement de ces douze différentes variétés d'arachide. Suivant un dispositif en blocs complets randomisés avec trois répétitions, les données collectées ont été analysées selon la méthode de l'analyse de la variance (ANOVA) au seuil de probabilité de 5\%. Le test de la plus petite différence significative (PPDS) avait été utilisé pour comparer les différents paramètres observés. Les résultats sur la nodulation indiquent que la variété ICGV 98541 est la meilleure et a donné un nombre de nodules supérieur aux autres. Quant au rendement, la variété JL 24 a donné une production supérieure aux autres suivie de la variété ICGV 98 541. A cet effet, la variété JL 24 et la variété ICGV 98541 peuvent être retenues et recommandées aux agriculteurs en RDC comme variétés de cultures associées en mettant un accent particulier sur la variété ICGV 98541 pour son efficacité à noduler et son rendement relativement élevé.

(C) 2013 International Formulae Group. All rights reserved.

Mots clés : Fixation de l'azote, production, cultures associées, RDC.

\section{INTRODUCTION}

L'agriculture des pays d'Afrique tropicale est caractérisée par sa faible productivité. En République Démocratique du Congo (RDC), les faibles rendements des cultures sont souvent expliqués par la pauvreté naturelle des sols en éléments nutritifs et dont le plus important est l'azote et la faible utilisation d'engrais (Wey et Obaton, 1978).

Dans le contexte actuel de crise alimentaire mondiale, la valorisation des cultures vivrières s'avère indispensable. En effet, La forte croissance démographique des 
dernières années entraîne une forte pression sur les ressources en terres cultivables et occasionne du même coup une augmentation de la demande en produits végétaux utiles à l'homme (Dugué et al., 2004a, 2004b; Bangata et al., 2013).

Cette forte pression sur le couvert végétal influence la capacité des sols à produire la biomasse nécessaire aux besoins d'une population de plus en plus nombreuse. Dans un tel contexte, la jachère qui était le moyen traditionnel de restauration de la fertilité des sols est moins pratiquée à cause de la forte demande en terres cultivables (Matokot et al., 1987; Dugué et al., 2004a, 2004b).

Face à cette situation, il est urgent de développer des techniques de fertilisation rationnelle, efficiente et accessible aux producteurs à moindres coûts et qui permettent d'augmenter la production par unité de surface tout en maintenant la fertilité des sols à long terme (Papy et Torre, 2003).

L'arachide (Arachis hypogaea L.) est présente dans les systèmes de culture et demeure, aujourd'hui pratiquement la seule légumineuse qui est utilisée couramment dans les pays en voie de développement comme c'est notamment le cas en RDC, pour les assolements villageois ou paysans et constitue une alternative pour améliorer la fertilité des sols et le rendement des cultures (Hamasselbé, 2008).

A cet effet, la variété Bayanzi produirait moins et présenterait un taux de nodulation inférieur à toutes les autres dans divers ensembles agro-écologiques congolais et répondrait encore mal lorsque les paysans sont en mesure de l'introduire dans la rotation et son rendement est plus bas.

Dans ce travail, nous avons déterminé l'abondance de la nodulation à 60 jours après le semis de douze variétés d'arachide dans les conditions naturelles de la Station de Ndjili Brasserie et évalué la production de différentes variétés en vue de la vulgarisation de leur exploitation en cultures associées ou dans le système de rotation au près des paysans cultivateurs de Kinshasa.

\section{MATERIEL ET METHODES Site expérimental}

L'expérimentation a été conduite à N'djili brasserie situé à environ 30 kilomètres du centre ville de Kinshasa (4²9' de latitude Sud et $15^{\circ} 23^{\prime}$ de longitude Est, $471,31 \mathrm{~m}$ d'altitude) en RDC. Les précédents culturaux étaient constitués d'une jachère dominée par Cynodon dactylon, Panicum repens et Cyperus rotundus. Les sols de Kinshasa, ce sont des sols à texture essentiellement sablonneuse. La faible capacité de rétention en eau de ces sols leur confère une utilisation marginale pour l'agriculture (Cisse et Vachaud, 1987). L'essai a été conduit du 14 Avril au 24 Juillet 2012 (saison culturale $\mathrm{B} / 2012$ ). La température pendant la période de l'essai a varié entre 25,3 et $27,7^{\circ} \mathrm{C}$.

Le climat est du type $\mathrm{Aw}_{4}$ (Köppen) avec deux saisons : l'une pluvieuse, va de fin octobre jusqu'au début du mois de Mai, l'autre étant sèche. La pluviométrie est de $1317 \mathrm{~mm}$ et l'humidité moyenne de $85 \%$ (Ndembo et al., 1987).

\section{Matériel végétal}

Douze variétés d'arachide utilisées au cours de notre expérimentation provenaient de trois sources : le Programme de légumineuse de l'Institut National pour l'Etude et la Recherche Agronomiques (INERA) de Mvuazi dans la province du Bas-Congo nous a fourni neuf variétés. Il s'agit de : ICGV-SM 96722; ICGV-SM 281; ICGV-SM 01506; ICGV-SM 99588; ICGV-SM 86021; ICGVSM 95530; ICGV-SM 95523; ICGV-SM 98541 et A1408. Ensuite, le Service National de Semences (SENASEM) par sa Direction de Kinshasa, nous a disposé deux variétés : JL 24 et $\mathrm{G} 17$ et enfin, la variété locale trouvée chez les paysans aux alentours de la Station appelée Bayanzi. 


\section{Méthodes}

L'essai a été conduit suivant un dispositif en blocs complets randomisés avec 3 répétitions. Chaque bloc, représentant une répétition, comporte 12 parcelles correspondant aux variétés étudiées, soit un total de 36 parcelles. Les parcelles avaient une superficie de $1 \mathrm{~m}^{2}$ soit $1 \mathrm{~m}$ de côté et chacune d'elle comptait au totale 36 plants. Le champ avait $65,6 \mathrm{~m}^{2}$ soit $16,40 \mathrm{~m}$ de long et $4 \mathrm{~m}$ de large. Deux blocs consécutifs étaient séparés par une allée de $0,5 \mathrm{~m}$, tandis que deux parcelles consécutives étaient distantes de 0,40 m. Le terrain a été labouré et les parcelles ont été tracées et piquetées avant le semis; ce dernier a été fait aux écartements de $20 \mathrm{~cm}$ dans tous les sens.

L'ouverture du terrain a été effectuée par le défrichement du 10 au 13 Avril 2012. Le semis en poquet avait été effectué le 14 Avril 2012 à $5 \mathrm{~cm}$ de profondeur, en raison de deux graines par poquet.

En ce qui concerne les techniques culturales, le démariage a été effectué deux semaines après le semis simultanément avec le regarnissage des vides. Ensuite, le sarclage était associé au binage pour améliorer l'aération. Enfin pour clôturer, le roulage a été effectué un mois après le semis à l'aide d'un petit tonneau ou fût vide afin de permettre à un bon nombre de gynophores d'être en contact avec le sol suivant la méthode recommandée par Wey et Obaton (1978).

Les données ont été enregistrées à partir d'un échantillon de 10 plants choisis au hasard par parcelle expérimentale. Les observations ont porté sur les paramètres végétatifs et les paramètres de production suivants : le Taux de levée (\%), le Diamètre au collet $(\mathrm{cm})$, la Hauteur des plants $(\mathrm{cm})$, Le nombre de nodules à 60 jours après le semis (le nombre de nodules était obtenu après déracinement de dix plants de bordure par parcelle), le rendement à l'ha (obtenu en extrapolant à partir du poids moyen des gousses fraîches, sèches et graines sèches par plante selon la formule : Le poids moyen des gousses fraîches, sèches et graines sèches $\mathrm{x}$ la densité à l'hectare. Les écartements étaient de $20 \mathrm{~cm} \mathrm{x} 20 \mathrm{~cm}$ et la densité était ainsi de 250.000 plantes à l'hectare.

\section{Analyse statistique}

Pour chaque variable étudiée (le taux de levée, le diamètre au collet, la hauteur et le nombre de nodules par plant, le rendement des gousses fraîches, le rendement des gousses sèches et le rendement des graines sèches à l'hectare), les données collectées ont été analysées selon la méthode de l'analyse de la variance (ANOVA) au seuil de probabilité de $5 \%$. Le test de la plus petite différence significative (PPDS) avait été utilisé pour comparer les résultats des différentes variétés d'arachide.

\section{RESULTATS}

\section{Paramètres végétatifs et la nodulation Levée des plants}

La levée des plants s'est étalée de 4 à 7 jours après semis et les résultats relatifs au taux de levée ont été évalués 9 jours après le semis. Ils indiquent que toutes les variétés utilisées sont de bonne qualité car leur pouvoir germinatif est supérieur $91 \%$.

\section{Le diamètre au collet, la taille et le nombre de nodules par plant de douze variétés.}

Les résultats de ces deux paramètres végétatifs et celui du nombre moyen de nodules par plant sont donnés dans le Tableau 1.

Le Tableau 1 montre que toutes les variétés ont de manière significative influencé la croissance en diamètre avec une forte croissance pour la variété ICGV 86 021, qui a donné un diamètre moyen au collet supérieur aux autres et la variété JL 24 a montré une faible croissance. L'analyse statistique au seuil de probabilité $5 \%$ a donné des différences (PPDS $=0,012$ ) entre les variétés en ce qui concerne la croissance en diamètre de ces différentes variétés. Quant à la croissance en hauteur, il ressort que toutes les variétés n'ont pas présenté la même hauteur. 
En effet, seules les variétés ICGV 95530 et ICGV 95523 ont montré une hauteur significativement élevée par rapport aux autres (PPDS $=4$ ), suivi des variétés ICGV 95588 et G 17. La variété ICGV 281 a présenté une courte taille de $12,8 \mathrm{~cm}$ correspondant à la limite inferieure selon les critères de Pollet (1984) et De Waele et Swaneverder (2001). En effet, pour certaines variétés et dans certaines conditions écologiques, elle varie entre 15 et $100 \mathrm{~cm}$.

En rapport avec les résultats relatifs au nombre des nodules par plant, l'analyse statistique montre que la variété ICGV 98541 a donné le nombre le plus élevé de nodules, soit 132 nodules en moyenne alors que le nombre le plus bas a été enregistré chez la variété locale Bayanzi qui a produit en moyenne 31 nodules. Le nombre moyen de nodules produits par la variété ICGV 98541 reste cependant supérieur avec ceux obtenus par Museu (2004) avec une moyenne de 118 nodules. L'analyse statistique a montré de différence significative (PPDS $=52,07$ ) entre les variétés.

Malgré ces différences remarquées, la présence des nodules sur ces différentes variétés prouvent que chaque variété sous étude a pu trouver une souche de rhizobium spécifique en mesure de la noduler dans le sol et qu'elles peuvent améliorer la nodulation même dans les sols très pauvres en azote tel que celui de notre essai.

\section{Paramètres de production}

Les résultats sur la production moyenne des gousses fraîches, sèches et graines sèches triées sont repris dans le Tableau 2.

Les résultats sur le rendement dans cette étude variaient de 3,5 à 5,1 tonnes. L'analyse statistique montre qu'aucune différence significative n'existe entre les variétés.

Tableau 1: Résultats relatifs au diamètre au collet, la taille et le nombre moyen de nodules par plan de douze variétés d'arachide en essai.

\begin{tabular}{lccc}
\hline Variété & $\begin{array}{c}\text { Diamètre moyen au } \\
\text { collet des plants }(\mathbf{c m}) \text { et } \\
\text { 土écart-type }\end{array}$ & $\begin{array}{c}\text { Hauteur moyenne } \\
\text { des plants (cm) et } \\
\text { 土écart-type }\end{array}$ & $\begin{array}{c}\text { Nombre moyen des } \\
\text { nodules par plant et } \\
\pm \text { écart-type }\end{array}$ \\
\hline Locale Bayanzi & $0,34 \pm 2,3 \mathrm{ab}$ & $13,3 \pm 6,12 \mathrm{bcdefg}$ & $31 \pm 3,7 \mathrm{bdf}$ \\
ICGV 96 722 & $0,36 \pm 2,6 \mathrm{ae}$ & $13,3 \pm 5,37 \mathrm{bcdef}$ & $50 \pm 4,6 \mathrm{bcd}$ \\
JL 24 & $0,31 \pm 2,42 \mathrm{bf}$ & $14,5 \pm 5,33 \mathrm{ae}$ & $46 \pm 4,4 \mathrm{bcd}$ \\
G 17 & $0,35 \pm 2,4 \mathrm{a}$ & $16 \pm 6,01 \mathrm{ag}$ & $47 \pm 4,34 \mathrm{bcd}$ \\
ICGV 281 & $0,39 \pm 2,3 \mathrm{ac}$ & $12,8 \pm 6,23 \mathrm{bcdef}$ & $60 \pm 3,21 \mathrm{bcd}$ \\
A 1408 & $0,35 \pm 2,26 \mathrm{af}$ & $14 \pm 5,76 \mathrm{af}$ & $59 \pm 3,7 \mathrm{bcd}$ \\
ICGV 01506 & $0,34 \pm 2,3 \mathrm{ab}$ & $15,4 \pm 5,49 \mathrm{ac}$ & $63 \pm 4,1 \mathrm{bcd}$ \\
ICGV 99 588 & $0,4 \pm 2,4 \mathrm{ac}$ & $16,2 \pm 5,76 \mathrm{ab}$ & $76 \pm 4,54 \mathrm{bcd}$ \\
ICGV 86 021 & $0,47 \pm 2,6 \mathrm{a}$ & $15,1 \pm 5,8 \mathrm{ad}$ & $76 \pm 4,47 \mathrm{bcde}$ \\
ICGV 95 530 & $0,46 \pm 2,62 \mathrm{a}$ & $17,8 \pm 6,3 \mathrm{a}$ & $92 \pm 4,7 \mathrm{ad}$ \\
ICGV 95 523 & $0,37 \pm 2,48 \mathrm{ad}$ & $17,7 \pm 6,21 \mathrm{a}$ & $93 \pm 3,23 \mathrm{ace}$ \\
ICGV 98 541 & $0,33 \pm 2,43 \mathrm{bcdef}$ & $15,4 \pm 5,2 \mathrm{ag}$ & $132 \pm 4,6 \mathrm{a}$ \\
\hline PPDS (5\%) & 0,012 & 4 & 52,07 \\
\hline \multicolumn{4}{c}{ Les chiffres dans les colonnes suivis de même lettre ne sont pas significativement différents selon le test de la Plus } \\
Petite Différence Significative (PPDS) à 5\% de probabilité.
\end{tabular}


Tableau 2 : Rendement estimé à l'ha en gousses fraîches, sèches et graines sèches triées de douze variétés d'arachide en essai sont repris dans les Tableaux 2.

\begin{tabular}{lccc}
\hline Variété & $\begin{array}{c}\text { Rendement moyen de } \\
\text { gousses fraîches } \\
(\mathbf{t} / \mathbf{h a})\end{array}$ & $\begin{array}{c}\text { Rendement moyen } \\
\text { de gousses sèche } \\
(\mathbf{t} / \mathbf{h a})\end{array}$ & $\begin{array}{c}\text { Rendement moyen en } \\
\text { graines sèches } \\
(\mathbf{t} / \mathbf{h a})\end{array}$ \\
\hline Locale Bayanzi & $3,7 \pm 1,2$ & $3,6 \pm 1,02$ & $1,6 \pm 1,23 \mathrm{a}$ \\
ICGV 96 722 & $3,5 \pm 1,5$ & $3,1 \pm 1,12$ & $1,5 \pm 1,3 \mathrm{a}$ \\
JL 24 & $5,1 \pm 1,2$ & $4,2 \pm 1,4$ & $2,9 \pm 1,54 \mathrm{a}$ \\
G 17 & $4 \pm 1,3$ & $2,3 \pm 1,2$ & $1,5 \pm, 12 \mathrm{a}$ \\
ICGV 281 & $3,9 \pm 2,1$ & $2,6 \pm 1,3$ & $1,6 \pm 1,41 \mathrm{a}$ \\
A 1408 & $4,1 \pm 1,4$ & $2,6 \pm 1,1$ & $1,75 \pm 1,34 \mathrm{a}$ \\
ICGV 01506 & $4,2 \pm 1,12$ & $3 \pm 1,03$ & $1,5 \pm 1,27 \mathrm{a}$ \\
ICGV 99 588 & $4 \pm 2,1$ & $2,9 \pm 1,5$ & $2 \pm 1,03 \mathrm{a}$ \\
ICGV 86 021 & $4,5 \pm 1,7$ & $2,9 \pm 1,4$ & $1,7 \pm 1,2 \mathrm{a}$ \\
ICGV 95 530 & $4,2 \pm 1,5$ & $3,1 \pm 1,58$ & $1,75 \pm 1,25 \mathrm{a}$ \\
ICGV 95 523 & $3,7 \pm 1,6$ & $2,6 \pm 1,3$ & $1,5 \pm 1,3 \mathrm{a}$ \\
ICGV 98 541 & $4,6 \pm 2,3$ & $3,6 \pm 1,2$ & $2,5 \pm 1,6 \mathrm{a}$ \\
\hline PPDS (5\%) & $\mathrm{N} . \mathrm{S}$ & $\mathrm{N} . \mathrm{S}$ & 1,9 \\
\hline
\end{tabular}

Les chiffres dans les colonnes suivis de même lettre ne sont pas significativement différents selon le test de la Plus Petite Différence Significative (PPDS) à 5\% de probabilité.

\section{DISCUSSION}

En comparant nos résultats avec ceux présentés par Jan de Graaff et al. (2011), nous remarquons que ces auteurs ont pu obtenir des valeurs plus élevées, respectivement pour JL 24, 12,5 tonnes d'arachide de gousses fraîches à l'ha contre 5,1 tonnes pour notre étude, 9,2 tonnes pour la variété G17, contre 3,97 tonnes, et la variété A 1408, 6,8 tonnes contre 4,1 tonnes.

Il est à noter que, nos résultats sont inférieurs à ceux obtenus par Mbukula (2003). Ces différences pourraient être dues soit par la richesse du sol en matières organiques ou la période de l'essai. En ce qui concerne le rendement moyen d'arachide en gousses sèches, l'analyse statistique a montré qu'il n'y a pas de différence significative entre les différentes variétés. Toutefois, la variété JL 24 confirme sa performance quant à la production suivie de la variété ICGV 98541.

Ces résultats, par rapport aux travaux antérieurs montrent que la variété JL24 est moins productive par rapport aux résultats présentés par Mbukula (2003), cependant elle reste supérieure, quant à sa production, par rapport aux résultats présentés par Affholder (1995), dont le rendement à l'hectare variait de 1,313 à 1,612 tonnes.

Au regard de ces résultats relatifs aux graines sèches figurant sur la colonne $3 \mathrm{du}$ Tableau 2, nous remarquons que la variété JL 24 apparait nettement supérieure aux autres dans notre essai soit 2,9 tonnes de graines d'arachide par hectare. Comparativement à Affholder (1995), on peut remarquer que le rendement en graines d'arachide peut varier de 900 à $1500 \mathrm{~kg}$ par hectare en milieu paysan.

$\mathrm{Au}$ Bas-Congo, en particulier à l'INERA-M'VUAZI (RDC), les résultats en graines sèches d'arachide présentés par Mbukula (2003) variaient de 3,7 à 5,2 tonnes par hectare. Ces résultats sont deux fois supérieures à ceux obtenus dans notre étude, soit un rendement de 1,5 à 2,9 tonnes par hectare.

Par ailleurs, tenant compte de paramètre nodulation, les résultats montrent que la variété ICGV 98541 est performante (132 $\pm 4,6$ nodules). Par contre, la variété JL 24 a montré un résultat supérieur aux autres 
en ce qui concerne le rendement $(2,9 \pm 1,54$ tonnes). A cet effet, il est donc impossible d'établir une probable corrélation entre les différentes variétés étudiées par ce que le meilleur rendement a été observé chez la variété JL 24 qui a présenté une nodulation faible (coefficient de corrélation de Pearson $\left.\mathrm{R}^{2}=0,057\right)$.

Sur le plan de la géologie, N'djili Brasserie appartient au système schistogréseux. L'horizon $A_{1}(0-20 \mathrm{~cm})$ possède une texture sablo-argileux, une structure grumeleuse et une consistance friable. Le sol est extrêmement acide $(\mathrm{pH}<4,5)$ lié à la présence de l'Al ${ }^{3+}$ (FERRISOL) (Ndembo et al., 1987). Il s'agit donc d'un sol pauvre, son amélioration exige l'association des cultures (Céréales et légumineuses).

En effet, de façon isolée, la monoculture des céréales est difficile dans un tel type de sol à cause de son extrême pauvreté en azote. La fixation symbiotique de l'azote par les bactéries de nodosité présente à cet effet, un intérêt certain car elle permettrait de garantir la qualité (teneur en protéines) et la quantité (productivité élevée) des produits en vue de garantir la sécurité alimentaire de la population (Venkateswarlu et al.,1991; Eldra et al., 2005).

\section{Conclusion}

Les résultats obtenus ont montré qu'en ce qui concerne les paramètres végétatifs, la variété ICGV 86021 a montré un résultat satisfaisant en ce qui concerne le diamètre au collet par rapport aux autres. Pour la hauteur moyenne, le meilleur résultat a été observé chez les variétés ICGV 95530 et ICGV 95 523. Quant à la nodulation, c'est la variété ICGV 98541 qui est restée performante en termes de nombre de nodules $(132 \pm 4,6)$.

Pour ce qui est du rendement à l'hectare, les résultats globaux obtenus sur la production après l'évaluation comparée des caractéristiques et performances des douze variétés d'arachide à travers cette étude, ont mis en exergue la supériorité de la variété JL 24 (2,9 $\pm 1,54$ tonnes $)$.
Cependant, compte tenu de l'importance que l'on doit accorder tant à la productivité qu'à la durabilité du sol, la variété à recommander serait ICGV 98541 car elle a nodulé plus que toutes les autres variétés et a présenté un rendement significativement égal à la variété JL $24(2,5 \pm 1,60$ VS 2,9 $\pm 1,54)$.

Les études visant l'évaluation de l'efficacité symbiotique, l'indice de la nodulation, ainsi que la quantité d'azote fixée par chaque variété aux différents endroits sont en cours en vue de déterminer la meilleure variété parmi les douze pouvant faire l'objet de la vulgarisation comme variété de culture adaptée aux exigences des agriculteurs du point de vue performance de nodulation.

\section{REFERENCES}

Affholder F. 1995. Effect of organic matter input on the water balance and yield of millet under tropical dryland condition. Field Crops Research., 41(2): 109-121.

Bangata BM, Ngbolua KN, Ekutsu E, KalonjiMbuyi A. 2013. Comportement de quelques lignées de riz NERICA en culture de bas-fond dans la région de Kinshasa, République Démocratique du Congo. Int. J. Biol. Chem. Sci., 7(1): 2532.

Cisse L, Vachaud G. 1987. Effet d'un amendement organique sur l'infiltration, les coefficients de transferts hydriques, et l'évaporation d'un sol sableux dégradé du Nord-Sénégal. Hydrologie Continentale, 2(1): 15-28.

De Waele, Swaneverder. 2001. Plantes oléifères. Arachide. In Agriculture en Afrique Tropicale. Raemaekers RH (éd). Direction Générale à la Coopération Internationale (DGCI): Bruxelles; 768785.

Dugué $\mathrm{P}$, Koné FR, Koné G, Akindes F. 2004a. Production agricole et élevage dans le centre du bassin cotonnier de Côte d'Ivoire. Cahiers Agricultures, 13(6): 504-509.

Dugué P, Vall E, Lecomte P, Klein HD, Rollin D. 2004b. Evolution des relations entre l'agriculture et l'élevage dans les 
savanes d'Afrique de l'Ouest et du Centre. Oléagineux, 11(4): 268-276.

Eldra PS, Linda RB, Diana WM. 2005. Biology (7th edn). Thomson Learning Academic Resource Center: Belmont, USA.

Hamasselbé A. 2008. La revalorisation de la filière arachide dans la zone soudanosahélienne du Nord Cameroun. Tropicultura, 26(4): 200-205.

Jan de Graaff, Kessler A, Jan Willem N. 2011. Agriculture and food security in selected countries in Sub-Saharan Africa: diversity in trends and opportunities. Food Security, 3(2): 195-213.

Matokot L, Mapangou-Divassa S, Delobel A. 1987. Evolution des populations de Caryedon serratus (O1.) (Coleoptera: Bruchidae) dans les stocks d'arachide au Congo. Agronomie Tropicale, 42(1): 6974.

Mbukula M. 2003. Etude comparative de quelques variétés d'arachide (Arachis hypogaea L.) de l'Icrisat dans les conditions écologiques de M'vuazi (Bas Congo). Mémoire, Faculté des Sciences Agronomiques, UNIKIN, Inédit, p. 34.

Museu E. 2004. L'influence des doses croissante du phosphore et la fixation symbiotique de l'azote de quelques légumineuses à graines en sol sableux de
Kinshasa. Mémoire, Faculté des Sciences Agronomiques, UNIKIN, Inédit, p.36.

Ndembo L, Masozera WR, Kabwika M, Makumbi MN. 1987. Evolution des sesquioxydes de fer et d'Aluminium dans un sol de Kimwenza. Rev. Zar. Sci. Nucl., 8(1): 96-107.

Papy F, Torre A. 2003. Quelles organisations territoriales pour concilier production agricole et gestion des ressources naturelles? (à partir de questions d'agronomie et d'économie). Études et Recherches sur les Systèmes Agraires et le Développement, 1(33): 151-169.

Pollet. 1984. Cultures Traditionnelles Africaines de Plein Champ. Edition MASSON \& Cie: Paris.

Venkateswarlu B, Maheswari M, Subba Reddy G. 1991. Relationship between nodulation, nitrogen fixation rate, $\mathrm{N}$ harvest index and kernel yield in different groundnut varieties under dryland conditions. Oléagineux, 46(6): 239-243.

Wey J, Obaton M. 1978. Incidence de quelques techniques culturales sur l'activité fixatrice d'azote et le rendement de l'arachide. Agronomie Tropicale, 33(2): 129-135. 